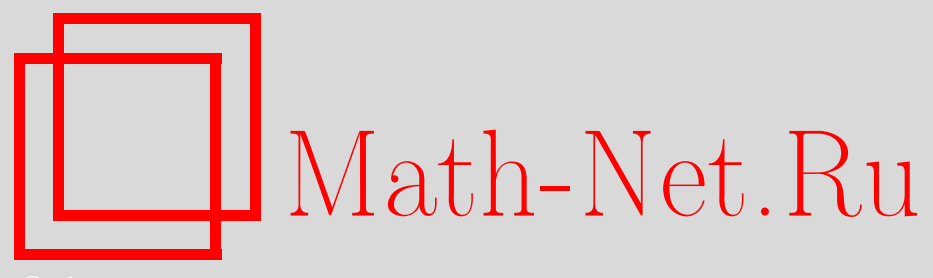

А. Романов, Юбилей теоремы Райского, Квант, 2019, номep 7, 10-12

DOI: https://doi.org/10.4213/kvant20190702

Использование Общероссийского математического портала Math-Net.Ru подразумевает, что вы прочитали и согласны с пользовательским соглашением http://www.mathnet.ru/rus/agreement

Параметры загрузки:

IP : 3.85 .7 .115

26 апреля 2023 г., 17:49:10

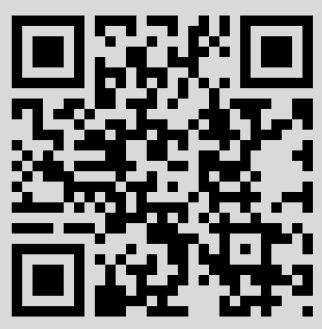




\section{Юбилей теоремы Райского}

A. POMAHOB

\section{0} ЛЕТ НАЗАД В ЖУРНАЛ «МАТЕМАтические заметки» была подана статья школьника Дмитрия Райского [1] с существенным продвижением в проблеме, поставленной Хадвигером [2]. Дмитрий доказал такую теорему:

При раскраске плоскости в три цвета ( разбиении на три непересекающиеся множества) всегда можно выбрать ивет, в котором реализуются все расстояния, т.е. для любого положительного $d$ найдутся две точки на расстоянии $d$, окрашенные в выбранный цвет.

Теорема Райского является заметным усилением доказанной в 1961 году братьями Мозерами теоремы о том, что при раскраске плоскости в три цвета найдутся две точки одного цвета на расстоянии 1. При подготовке статьи [1] к печати теорема была обобщена на $N$-мерное пространство и существенно отредактирована. В результате работа школьника оказалась трудночитаемой и недоступной для юных математиков. Здесь я постарался воспроизвести первоначальный вариант решения. Я благодарен Н.Н.Константинову, который в 1971 году рассказал мне доказательство теоремы Райского. Константинов помогал Дмитрию в подготовке статьи и без его участия публикация вряд ли была бы возможна.

\section{Вспомогательные леммы}

Пусть на плоскости задана фигура из $n$ различных точек - $n$-точечник. Выберем одну из этих точек и назовем $n$-пучком набор векторов, выходящих из этой точки ко всем точкам $n$-точечника (один из векторов $n$-пучка - нуль-вектор). Определим произведение $n$-пучка и $k$-пучка как пучок векторов $a+c$, где вектор $a$ берется из $n$-пучка, а $c$ - из $k$-пучка.

DOI: https://doi.org/10.4213/kvant20190702
Определенное таким образом произведение содержит не более чем $n \cdot k$ различных векторов, так как суммы векторов для разных пар могут и совпадать.

Лемма 1. Если мы рассмотрим повороты k-пучка на произвольный угол, то лишь для конечного числа углов поворота произведение фиксированного п-пучка и повернутого $k$-пучка будет содержать менее чем $n \cdot k$ различных векторов.

Действительно, выбрать два различных вектора $a, b$ из $n$-пучка и два различных вектора $c, d$ из $k$-пучка можно лишь конечным числом способов. Для каждой такой четверки векторов рассмотрим повороты $k$-пучка на угол $\alpha$ и сравним суммы векторов $a+c_{\alpha}$ и $b+d_{\alpha}$. Так как окружности пересекаются не более чем в двух точках, не более двух углов $\alpha$ могут дать равенство этих сумм, что и доказывает лемму 1.

Лемма 2. Если произведение п-пучка и $k$-пучка содержит $n \cdot k$ различных векторов, то $n \cdot k$ концов этих векторов образуют п фигур, полученных параллельным переносом $k$-точечника, и $k$ фигур, полу-

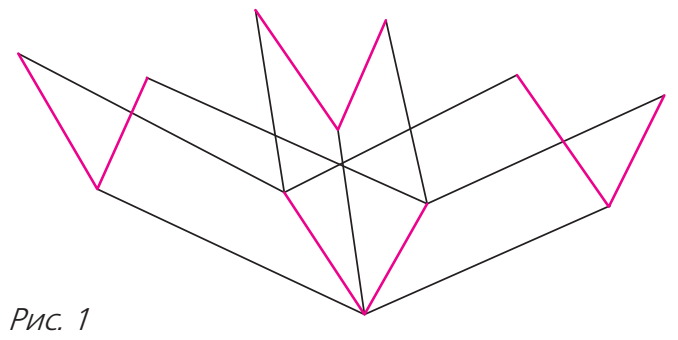

ченных параллельным переносом п-точечника (рис.1).

\section{Доказательство теоремы}

Предположим, что утверждение неверно и существует раскраска плоскости в три цвета, для которой нельзя выбрать цвет, в котором реализуются все расстояния. Тогда для этой раскраски у каждого цвета есть 
расстояние, которого не может быть между двумя точками это цвета.

Рассмотрим 7-точечник, предложенный братьями Мозерами, который Райский назвал «зондом» (рис.2). Все нарисованные отрезки имеют длину 1. Простым перебором можно убедиться, что если из

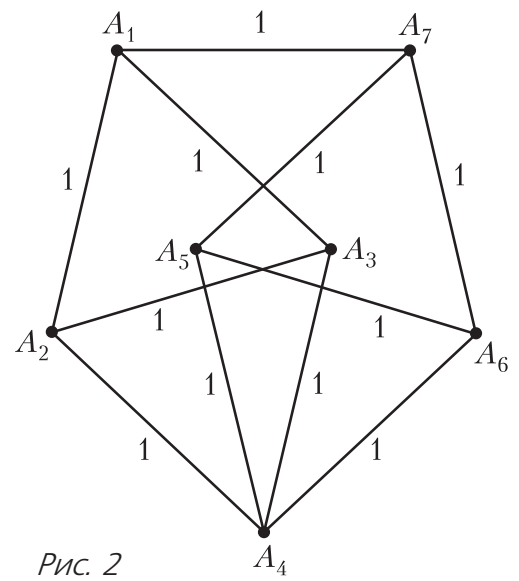

этих семи точек выбрать любые три, то найдутся две выбранные точки, соединенные отрезком. Из этого сразу следует теорема Мозеров.

У нас для каждого цвета будет свой 7-точечник-зонд, в котором вместо 1 будет отсутствующее в этом цвете расстояние. Выберем произвольную точку плоскости и все векторы пучков будем считать выходящими из этой точки. Пронумеруем цвета произвольно и рассмотрим зонд для цвета 1. В каждом из трех 7-точечников-зондов выберем одну из 7 точек, например $A_{4}$. Тогда для каждого зонда определен свой 7-пучок. По лемме 1 повернем 7-точечник-зонд для цвета 2 на такой угол, чтобы произведение 7 -пучков 1 и 2 содержало $7^{2}$ различных векторов (было 49-пучком), а 7-точечник-зонд для цвета 3 повернем на такой угол, чтобы произведение 49-пучка и этого 7-пучка содержало $7^{3}$ различных векторов. По лемме 2, концы этих $7^{3}$ векторов $-7^{3}$ различных точек на плоскости - образуют 49 зондов для цвета 3, они же образуют 49 зондов для цвета 2 и 49 зондов для цвета 1. Для завершения доказательства достаточно заметить, что в любом зонде для цвета 1 из 7 точек не более двух цвета 1, в любом зонде для цвета 2 из 7 точек не более двух цвета 2, а в любом зонде для цвета 3 из 7 точек не более двух цвета 3.

Тем самым, не более $2 \cdot 7^{2}$ точек может быть окрашено в каждый цвет, всего не более $6 \cdot 7^{2}$ точек из $7^{3}$ может быть окрашено.

Доказательство завершено.

\section{Многомерный случай}

В статье Райского приведено доказательство теоремы для $n$-мерного пространства:

Пусть множества $A_{1}, A_{2}, \ldots, A_{n+1}$ образуют покрытие $n$-мерного евклидова пространства $\mathbb{R}^{n}$ ( $\left.n>1\right) ;$ тогда среди них найдется такое множество $A_{i}$, что для всякого $d>0$ найдется пара точек из этого множества, расстояние между которыми равно $d$.

Для доказательства теоремы в таком виде не требуется новых идей, кроме конструирования зонда в $\mathbb{R}^{n}$ и доказательства леммы 1. Чтобы не жертвовать доступностью изложения, рассмотрим случай трехмерного пространства, для больших измерений трудности носят чисто технический характер. (Заметим, что в статье Райского доказательство существенно отредактировано, здесь я постарался воспроизвести первоначальный вариант.)

Построим зонд для $\mathbb{R}^{3}$. Веретеном Райский назвал 5-точечник из четырех вершин правильного тетраэдра со стороной $d$ и еще одной точки $b$, симметричной одной из его вершин - вершине $a$ - относительно грани (рис.3). Зондом он назвал 9-точечник, полученный из двух веретен, совмещенных вершинами $a$ и повернутых так, чтобы расстояние между точками $b$ этих веретен тоже было равно $d$ (рис.4).

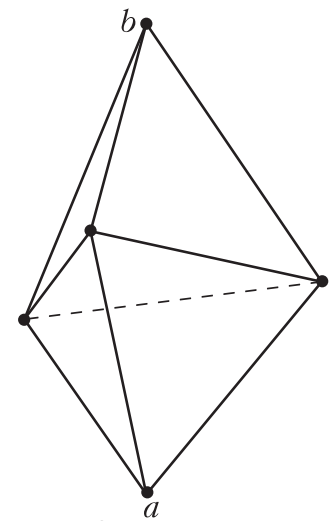

Рис. 3 


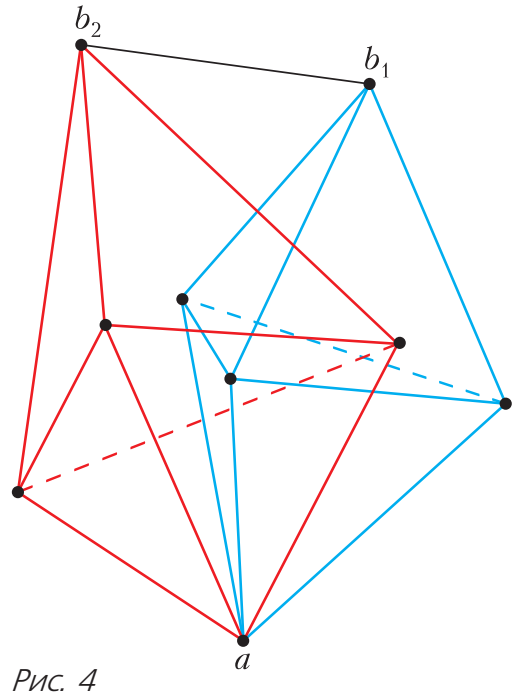

Очевидно, что такой поворот веретен существует.

Доказательство леммы 1 нуждается в незначительной модернизации. Для заданного $n$-точечника выберем плоскость в $\mathbb{R}^{3}$ так, чтобы проекция $n$-точечника на эту плоскость содержала $n$ различных точек. Выберем исходное положение (поворот) $k$-точечника относительно этой плоскости, чтобы его проекция содержала $k$ различных точек. На плоскости получились $n$ пучок и $k$-пучок, для которых справедлива лемма 1. Рассмотрим поворот исходного $k$ пучка вокруг прямой, перпендикулярной выбранной плоскости. Проекция на плоскость содержит $n \cdot k$ различных векторов, что и доказывает лемму 1 для $\mathbb{R}^{3}$. Остальная часть доказательства проводится без изменений.

\section{Оптимизация доказательства теоремы Райского}

Предложенное доказательство не использует идей, отсутствующих в первоначальном варианте Райского. Оказывается, можно отказаться от рассмотрения поворотов, делающих точки не совпадающими (лемма 1). Вместо этого можно каждому вектору произведения $n$-пучка и $k$-пучка приписать кратность - количество вариантов выбора вектора из $n$-пучка и вектора из $k$-пучка, для которых суммы векторов совпадают .
Лемма 2 меняется незначительно. Произведение $n$-пучка и $k$-пучка содержит $n \cdot k$ векторов с учетом кратности, и $n \cdot k$ концов этих векторов образуют $n$ фигур, полученных параллельным переносом $k$-точечника, и $k$ фигур, полученных параллельным переносом $n$-точечника. Каждая точка произведения пучков встречается в этих фигурах столько раз, какова кратность точки.

При таком подходе многомерный случай отличается от двумерного только конструкцией зонда.

\section{Оценка сверху}

До публикации статьи Райского была известна раскраска плоскости в 7 цветов, в которой любые две точки на расстоянии 1 раскрашены в разные цвета. Эта раскраска основана на разбиении плоскости на равные правильные шестиугольники.

С.Б.Стечкин заметил (и с разрешения Райского вставил в статью), что плоскость можно разбить на 6 таких множеств, чтобы на каждом из них реализовались не все расстояния. Элемент конструкции состоит из 4 правильных шестиугольников и 8 правильных треугольников. Путем параллельных переносов этим элементом мостится вся плоскость, как показано на ри-

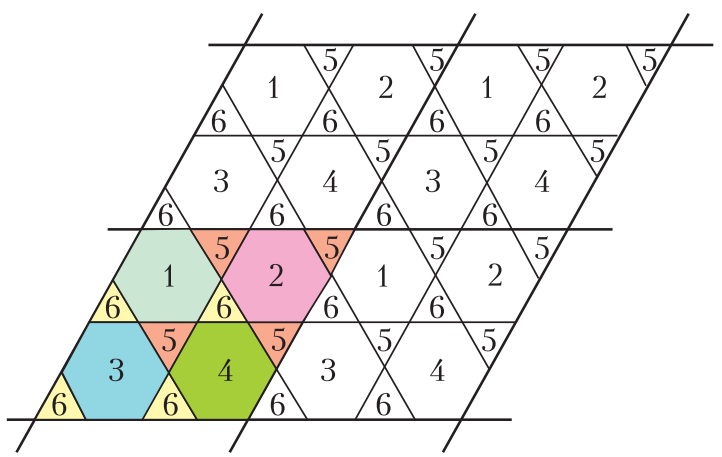

Рис. 5

сунке 5. Все треугольники являются открытыми, а нижние и правые вершины шестиугольников не принадлежат этим фигурам. Если принять сторону треугольника за 1 , то расстояние $d_{1}=2$ не реализуется на множествах $A_{1}, \ldots, A_{4}$ одинаково

(Продолжение см. на с. 23) 
Подведем итог, еще раз сформулировав основные свойства данной конфигурации.

1. Точки $K, L, P, Q$ образуют ортоцентрическую четверку.

2. $K P=Q L ;$ углы четырехугольника $K L P Q$ равны $45^{\circ}$ или $180^{\circ}+45^{\circ}$.

3. Точка $F=K L \cap P Q-$ середина отрезка $X Y$.

4. Точка $E=L Q \cap K P-$ проекция $C_{1}$ на прямую $I H$.

5. Точка $D=K Q \cap L P$ лежит на прямой $A B$.

6. Прямая $P Q$ проходит через $C$.

В завершение скажем, что на XXII Кубке памяти А.Н.Колмогорова одиннадцатиклассник Владимир Петров из СанктПетербурга придумал другое решение исходной задачи, позволяющее обнаружить еще ряд свойств. Приведем план этого решения.

Так же, как и ранее, заметим перпендикулярное подобие четырехугольников $K I L C_{1}$ и $Q C_{1} P H$, откуда $P Q \perp K L$.
Пусть $E^{\prime}$ - проекция $C_{1}$ на $I H$ (по факту мы знаем, что $E^{\prime}=E$ ), тогда $E^{\prime}$ - центр поворотной гомотетии (с углом поворота $90^{\circ}$ ), переводящей $\overrightarrow{H C_{1}}$ в $\overrightarrow{C_{1} I}$, а значит, $Q C_{1} P H$ в $K I L C_{1}$. Из поворотной гомотетии $E^{\prime} P \perp E^{\prime} L$. Теперь достаточно доказать, что $\angle K E^{\prime} L=90^{\circ}$, т.е. точки $K, C_{1}$, $E^{\prime}, L$ лежат на одной окружности.

При инверсии относительно вписанной окружности $E^{\prime}$ переходит в $H$. Отметим точку $T$ на продолжении $H C$ за точку $C$ такую, что $C T=C B_{1}=C A_{1}$. Тогда для завершения решения достаточно доказать, что образы $K^{\prime}, L^{\prime}, H$ и $C_{1}$ точек $K, L, E^{\prime}$ и $C_{1}$ лежат на окружности с диаметром $C_{1} T$.

\section{Упражнения}

8. Докажите это.

9. Пусть окружность с центром $C$ и радиусом $C A_{1}=C B_{1}$ пересекает $C H$ в точке $G$. Докажите, что $F$ - середина $G C_{1}$.

\section{Юбилей теоремы Райского}

\section{(Начало см. на с. 10)}

отмеченных шестиугольников, а расстояние $d_{2}=1$ не реализуется на множествах $A_{5}$ и $A_{6}$ одинаково отмеченных треугольников.

\section{Ожидаемые продвижения}

Вопрос о том, можно ли аналогичным образом разбить плоскость на 4 или 5 множеств, остается открытым. Но недавнее доказательство [3] теоремы о том, что при раскраске плоскости в четыре цвета найдутся две точки одного цвета на расстоянии 1 , дает основание надеяться, что для четырех цветов утверждение теоремы Райского тоже верно. Достаточно найти дистанционный граф (т.е. граф, вершины которого - точки на плоскости, а ребрами соединены только пары вершин на расстоянии 1) из $n$ вершин, для которого, если выбрать не менее $n / 4$ вершин, какая-то пара выбранных вершин обязательно будет соединена ребром. Предлагаю читателям убедиться, что если в качестве зонда выбрать такой граф, то конструкция Райского доказывает утверждение для четырех цветов, а математикам-программистам - заняться увлекательной задачей поиска подходящего графа. Сейчас известно много графов, доказывающих теорему о четыpex цветах, но, возможно, не каждый граф, найденный для этой цели, обладает нужным нам свойством.

\section{Литература}

1. Д.Е.Райский. Реализация всех расстояний при разбиении пространства $\mathbb{R}^{n}$ на $n+1$ часть. Математические заметки, т.7, №3, 1970, с.319323.

2. Г.Хадвигер, Г.Дебруннер. Комбинаторная геометрия плоскости. - М., 1965.

3. А.Райгородский, В.Воронов, А.Савватеев, Прорыв в задаче о раскраске плоскости. - «Квант», 2018, №11. 